\title{
EDITORIAL
}

\section{Preparedness to Respond to the Ever-increasing Cancer Cases}

\author{
Abraham Haileamlak, MD, Professor of Pediatrics and Child Health
}

DOI: http://dx.doi.org/10.4314/ejhs.v25i4.1

Cancer is on the rise globally and posing significant risk in many low- and middle-income countries. Worldwide, over twenty million people, majority in developing countries, are living with cancer. Death tolls from cancer globally surpass those from infectious diseases like AIDS, tuberculosis and malaria. This is because of the fact that strong preventive and therapeutic measures are taken those infectious diseases, but no or little effort on cancer. However, thru current knowledge, over a third of cancer that occurs every year could be prevented and another third could be detected early and treated effectively. For the remaining third, improving the quality of life of cancer patients and their families is the other arm cancer care. Many low and middle income countries started national programs to face the rising challenge.

Cognizant this, the Ethiopian government while continuing exerting its effort to tackle communicable diseases, it also turned its face to non-communicable diseases in general and cancer in particular. The move to address the ever increasing cancer cases is in various ways;

1. Policy and guidelines: most of the recently developed documents including the recently revised health policy, visioning document and health service transformation plan considered cancer in their content. Based on these premises, strategy on how to prevent and treat NCDs including cancer is developed and management guidelines for continuum of care are on development process. Appreciating what is done until now, finalizing and detailing the guidelines with clear scope of practice and continuum of care is further task to do.
2. Leadership: the Federal Ministry of Health has set structure that can work on NCDs in general and cancer in particular. Under the directorate for disease prevention and control, an NCD unit is formed where cancer has a focal person. Regional health bureaus also have similar arrangements. However, the link between regional health bureaus and university hospitals should be strengthened for better care and flow of information and resources.

3. Treatment and care facility: the federal ministry of health identified six university hospitals where a better chemo and radiotherapy centers are built. Most the identified centers have started the construction to start functioning in a year time. The equipment to be purchased and commissioned centrally. Several efforts are made to harmonize the design of the buildings and the pace of construction with the type of equipment to be purchased. Though early, systems need to be set to continuous maintenance of the machines and proper disposal of leftovers.

4. Human power development: with partners and the identified university hospitals, trainees are identified. Some of them started the training in country while some goes abroad and some other waiting. Building local capacity to train oncologists, oncology nurses, pharmacists, pathologists and transfusion medicine specialists is a must to feel the gap.

5. Supplies: The pharmaceuticals fund and supply Agency (PFSA) is mandated to provide the drugs, reagents and other supplies. I can imagine that continuity of supplies provision, sustainability of the service and affordability could be subsequent challenges. 
The current issue (Vol. 25, No 4), the fourth issue of the 25th year anniversary of EJHS contains nine original articles on varied subjects, one review and five case reports focusing on different areas. Two of the original researches published in this issue are on cancer in low and middle income countries; one on risk factors for cervical cancer whiles the other on treatment of breast cancer. I invite readers to read through these articles and appreciate or utilize the contents. I also encourage readers to forward comments and suggestions. 